Meta

Journal des traducteurs

Translators' Journal

\title{
Pensée, culture et message
}

\section{Roland Haumont}

Volume 12, numéro 3, septembre 1967

URI : https://id.erudit.org/iderudit/003040ar

DOI : https://doi.org/10.7202/003040ar

Aller au sommaire du numéro

Éditeur(s)

Les Presses de l'Université de Montréal

ISSN

0026-0452 (imprimé)

1492-1421 (numérique)

Découvrir la revue

Citer cet article

Haumont, R. (1967). Pensée, culture et message. Meta, 12(3), 75-80.

https://doi.org/10.7202/003040ar

Ce document est protégé par la loi sur le droit d'auteur. L’utilisation des services d'Érudit (y compris la reproduction) est assujettie à sa politique d'utilisation que vous pouvez consulter en ligne.

https://apropos.erudit.org/fr/usagers/politique-dutilisation/
Cet article est diffusé et préservé par Érudit.

Érudit est un consortium interuniversitaire sans but lucratif composé de l’Université de Montréal, l'Université Laval et l'Université du Québec à Montréal. Il a pour mission la promotion et la valorisation de la recherche. https://www.erudit.org/fr/ 


\section{Pensée, culture et message}

Depuis quelque temps, il n'est pas de semaine sans qu'un quotidien ou l'autre ne nous informe qu'on accuse le Ministère de l'Éducation de vouloir, par le truchement de ses technocrates, "anglifier» notre génération montante grâce à des manuels traduits de l'américain.

Présentée telle quelle, cette accusation semble vraie en apparence. Mais si on y regarde d'un peu plus près, on s'aperçoit vite que toute l'accusation est posée en porte à faux et que le problème est tout autre que celui que les accusateurs voudraient soulever.

En effet, si la traduction était pernicieuse en soi, je suis fermement convaincu que notre civilisation serait encore en train de patauger dans un magma latino-grec, puisque la plupart d'entre nous ont été soumis aux philosophies grecques et latines par l'intermédiaire des traductions. Quels sont ceux d'entre nous qui ont tiré la « substantifique moelle » de Platon, Aristote et Virgile «dans le texte» comme on dit? Si à cela j'ajoute les œuvres de Gœthe, Schiller, Petofi, Dante, Cervantes, Tolstoï, Dostoïevsky, Gogol, Ibsen, etc., il s'ensuit, en maintenant la thèse des protestataires, que notre enseignement devrait comporter l'étude d'un bon nombre de langues étrangères avant de pouvoir aborder l'étude des humanités. À moins que ces messieurs ne prétendent que l'étude d'une seule culture (oubliant ses tenants et encore plus ses aboutissants) suffit à donner au jeune qui verra le $\mathrm{xxI}^{\mathrm{e}}$ siècle une Weltanschauung à l'échelle de son époque.

La traduction ou plutôt la mauvaise traduction de manuels étrangers peut être pernicieuse si cette traduction fait violence à la réalité culturelle avec laquelle elle devrait cadrer pour être le véhicule valable d'une certaine donnée académique. La traduction n'est-elle pas, au fond, que la réexposition d'un message dans une langue autre que la langue de l'énoncé original ?

Ceci nous amène à reprendre un certain nombre de postulats qui devraient servir de base à la pensée linguistique de tout traducteur digne de ce nom.

Lorsque, sous l'impulsion de Ferdinand de Saussure, la linguistique historique est entrée dans une voie synchronique, annoncée dès 1877 par le Danois Verner, nous voyons apparaître cette fameuse dichotomie langue-parole qui hypnotisera beaucoup de praticiens de la langue. Il faudra attendre la venue de Gustave Guillaume pour que l'on pense sérieusement au fait que le message relève de trois niveaux interdépendants: la pensée, la langue, la parole; et surtout que tout message est «une alliance de l'observation fine du concret et de la réflexion abstraite profonde ${ }^{\mathbf{1}}$.

Nous sommes ici en état constaté mais non encore expliqué. Nous percevons intuitivement l'interdépendance qui existe entre les trois niveaux mentionnés mais nous ne sommes pas encore très éclairés sur la forme qu'elle peut prendre.

1. G. Guillaume, la Langue est-elle ou n'est-elle pas un système?, Québec, Presses de 1'Université Laval, 1952, p. 4. 
Dans le cas de la traduction, il est évident que cette dernière devra fonctionner aux trois niveaux, car un inventaire, même rapide, permet de révéler les écueils sur lesquels on peut trébucher en oubliant un seul de ces niveaux, surtout le premier.

Comme point de départ, nous avons le message original, c'est-à-dire l'enveloppe signifiante concrète d'un abstrait signifié. Cette enveloppe relève de deux niveaux, la langue et la parole, mais découle d'un autre, la pensée. Cependant nous allons voir que la pensée se trouve à son tour influencée par des schèmes sur lesquels elle doit s'ordonner pour devenir message. Ces schèmes sont fixés et figés par les habitudes socio-culturelles du milieu qui utilise une langue donnée. Il est évident que des cultures procédant de courants différents ne produiront pas des parallélismes linguistiques et c'est ici que le traducteur se doit d'y penser. Il doit cesser de faire de la technique, c'est-à-dire de prendre une expression figée, apprise par cour, sans bien comprendre le mécanisme qui l'amène à la remplacer par l'expression figée correspondante dans la langue de départ (apprise par cœur également).

Prenons comme exemple une série d'expressions anglaises et françaises qui recouvrent la même réalité:

1. He tiptoed down the stairs.

2. He ran up the street.

Il descendit l'escalier sur la pointe des pieds.

3. He flew across the Atlantic.

Il remonta la rue en courant.

Il traversa l'Atlantique en avion.

Dans chaque cas, nous voyons que la réalité nous est présentée dans un ordre différent selon la langue. Vous pourriez me dire que cet ordre nous est imposé par la syntaxe de chaque langue, cela est vrai. Mais il est tout aussi vrai que cet ordre imposé par la grammaire n'est pas sans avoir entraîné, après plusieurs siècles d'utilisation, une certaine façon de voir les choses.

Examinons plus attentivement les trois exemples ci-dessus; voyons selon quels schèmes intellectuels les idées nous sont présentées suivant que l'on s'exprime en anglais ou en français.

L'anglais nous offre l'ordre suivant:

A) - la façon dont l'action est accomplie;

B) les limites ou la direction de cette action;

C) l'endroit où elle s'accomplit.

Le français nous offre l'ordre ci-dessous:

A) ce que l'action a accompli;

B) où cette action a eu lieu;

C) comment elle s'est accomplie.

Ainsi, pour passer de l'anglais au français en se basant uniquement sur cet ordre d'apparition des idées, on obtient:

$$
\text { ANGLAIS ABC }=\text { FRANÇAIS BCA }
$$

De cette constatation, nous pouvons émettre un premier postulat que d'autres exemples du même genre viendraient confirmer: l'anglais a tendance à être « phénoménal $»^{2}$ tandis que, devant la même réalité, le français aurait tendance à être conclusif.

2. «Phénoménal » est utilisé ici dans le sens d'attaché principalement au phénomène dépeint, sans accorder d'importance aux causes ou conclusions. 
Cette attitude mentale est-elle fortuite et uniquement attachée au genre d'expressions citées plus haut? Essayons de voir si, dans un autre domaine où la grammaire n'intervient plus, nous pouvons retrouver la même tendance générale. Le vocabulaire des outils et des appareils simples devrait être assez éloquent à ce sujet.

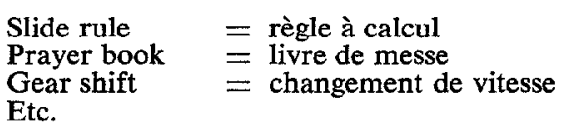

Ici encore nous voyons que l'anglais limite sa description à l'action accomplie sur un objet particulier tandis que le français va directement à l'objet général ou mieux encore, au résultat obtenu (ce qui est frappant dans le cas de règle à calcul).

Nous avons maintenant la certitude que la traduction du message apparent ne traduit pas la pensée à coup sûr, car, en passant d'une langue à l'autre, cette pensée change de cheminement et modifie de ce fait l'ordre d'accrochage de ses constituants. Il est évident que l'appareil qui, pour l'Anglais, sera décrit comme ayant un effet sur les pignons aura un nom concret (shift), tandis que ce déplacement de pignons aboutit à une modification de vitesse pour un Français et, de ce fait, recevra une appellation abstraite (changement).

Les messages succincts et quasi impératifs des panneaux de signalisation devraient obéir à la même règle, si nous sommes sur la bonne voie.

1. Men at work

2. No parking between signs.

Travaux en cours.

3. Slippery when wet.

Zone de stationnement interdit.

Chaussée glissante par temps humide (de pluie).

Ici encore, nous constatons que le locuteur anglophone a un point de vue totalement différent de celui d'un locuteur francophone voulant exprimer la même réalité.

Dans le premier cas, l'anglophone pense à ceux qui travaillent derrière la barrière jaune alors que le francophone pense à ce qui pourrait arriver à ceux qui dépassent la barrière avec leurs automobiles.

Le deuxième cas varie quant à la description de l'endroit impliqué par le message, l'anglophone nous en donne les limites tandis que le français le qualifie dans son ensemble.

Finalement pour le troisième cas nous constatons que l'anglais nous décrit le phénomène, sans plus. Le français pousse son analyse un peu plus loin (une espèce d'attitude concluante à rebours).

Nous voyons donc que l'anglais se limite souvent à la description «littérale 》 du phénomène, alors que le français cherche à pousser plus loin son analyse et à insérer le phénomène dans quelque chose de plus général.

Ce très bref tour d'horizon nous a bien montré que la pensée anglophone ne suit pas le même chemin que la pensée française. L'anglais a ainsi l'air de nous présenter une réalité toute différente. L'aspect «phénoménal » de l'anglais semble se préciser, alors que les tendances « analytiques » du français se confirment, tout au mooins dans les expressions ou syntagmes figés; cela doit être tout aussi vrai pour ce que l'on pourrait appeler la langue individuelle (si cela peut être), celle où les expressions préétablies n'existent pas.

Voilà le domaine où se concentrent les grands problèmes du traducteur, les 
problèmes qui, s'ils sont résolus, feront de l'œuvre traduite une œuvre d'art, une œuvre sur laquelle on ne portera pas le sempiternel jugement: "Ça sent la traduction ».

En somme, le traducteur doit éviter le trajet exposé dans le schéma ci-dessous:

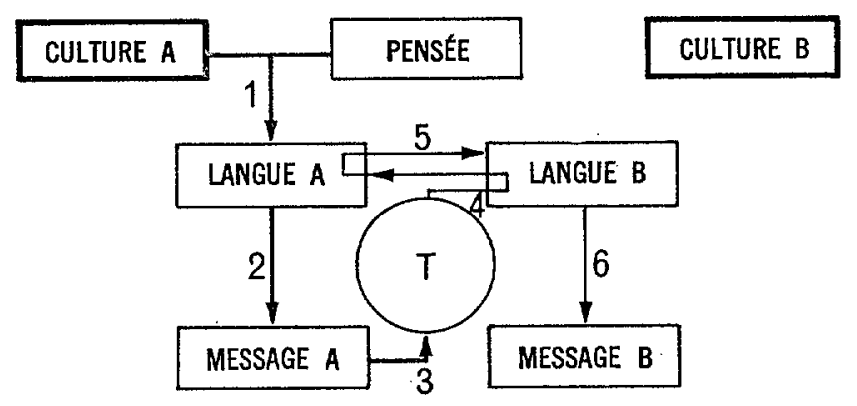

Dans ce schème, le traducteur reçoit le message $\mathrm{A}$ dans la langue de départ (en 3) après que celui-ci a été conçu par la pensée et la culture $\mathrm{A}$ et réalisé grâce aux options et servitudes de la langue $A$.

Si le traducteur se limite à un va-et-vient entre les langues $\mathrm{A}$ et $\mathrm{B}$, grâce aux dictionnaires, lexiques, etc. (en 5), il aboutira (en 6) à un message $\mathrm{B}$ qui sera un mélange de langue $\mathrm{B}$ et de culture $\mathrm{A}$; la culture $\mathrm{B}$ est totalement ignorée et tout travail peut se résumer par l'égalité apparente suivante:

$$
\text { MESSAGE B = LANGUE B + CULTURE A }
$$

Le traducteur doit approfondir les grands courants par lesquels sont agies les cultures des langues dans lesquelles il travaille et suivre en fait un schéma qui, dans ses grandes lignes, doit correspondre à celui-ci:

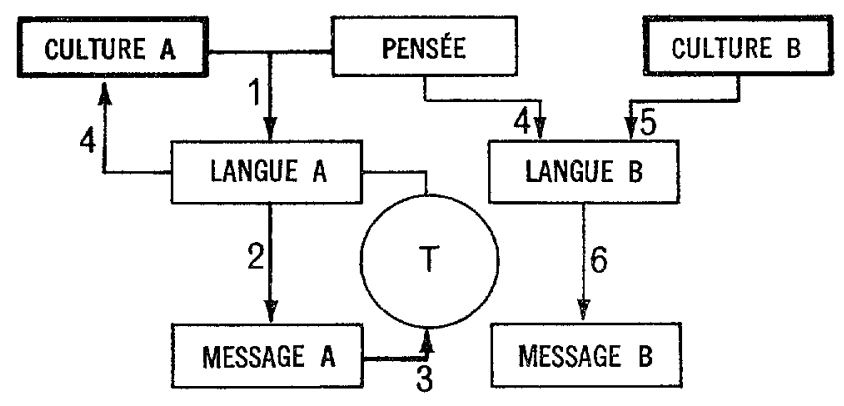

C'est-à-dire que, recevant le message $\mathrm{A}$ comme dans le schème précédent, il doit, par l'intermédiaire de la langue $\mathrm{A}$, le déshabiller de son contenu culturel et atteindre une pensée culturellement neutre. Cette pensée peut alors être colorée par tout ce qui est propre à la culture B (en 5), et par l'intermédiaire de la langue B, qui entre en jeu pour la première fois, aboutir à un message B. Cette fois, il y a parallélisme de démarches intellectuelles entre la réalisation des messages $\mathrm{A}$ et $\mathrm{B}$ :

Message a (LANGue a + Culture A) $\equiv$ Message B (LANGUe B + CUlture B) Notons également, avant de passer à un autre point, que le deuxième schème ne 
cumporte pas plus de démarches que le premier (six dans chaque cas). J'admets que certaines soient plus longues, en particulier celle qui porte le numéro 4 dans le deuxième schème. L'art du traducteur correspond, en fait, au talent qu'il mettra à effectuer ce déshabillage du message $\mathrm{A}$ original et à son choix et dosage des équivalences entre les cultures A et B tout en évitant ce que l'on pourrait appeler les « surtraductions».

Si l'on compare bien les deux schèmes, il devient évident que le second est le seul qui soit acceptable. Certaines des démarches qu'il implique sont longues et ardues, mais dans la traduction comme dans tout art, il n'y a pas de raccourci. Toute traduction digne de ce nom ne peut être «instantanée».

De ce que je viens d'écrire, il devient évident que le temps est bien révolu où tout un chacun pouvait se baptiser traducteur, s'il possédait quelques dictionnaires. Il faut que le traducteur d'aujourd'hui possède une solide culture dans chacune des langues dans lesquelles il veut ouvrer. Sa formation, sans pour cela être universitaire, se place au niveau de ce qu'il est convenu d'appeler "professionnel $»^{3}$. Comme tel, il devrait avoir droit au même respect que celui dont jouissent les autres professions libérales. Imaginez un client du traducteur critiquant et discutant le bien-fondé du diagnostic ou de l'ordonnance de son médecin ? Pourtant, il le fait pour le traducteur et bien souvent même en ne connaissant qu'incomplètement la langue de départ.

Ce désir dictatorial de l'ignorant linguiste est surtout évident dans le domaine de la traduction publicitaire où les perles sont si abondantes que la plupart d'entre nous ont cessé de les collectionner. Si un jeu de mots est passable dans une langue, il y a mille chances contre une qu'il soit incompréhensible dans l'autre. Cornme exemple, je citerai d'abord le slogan d'une brasserie britannique: $X \ldots$, the Brewery who put ale in Wales. Essayez de traduire cela en lui conservant toute sa saveur; si par hasard vous y arriviez, essayez-vous sur les deux suivants: Sweets minted by $X \ldots$... Shell qu'on aime ... Là, je crois que vous abandonnerez.

J'ai fait observer qu'à mon avis, si les manuels (en grande partie des manuels de sciences) sont mal traduits, ils sont pernicieux. En physique par exemple, la loi d'Ohm nous permet de mesurer et de prévoir certains phénomènes naturels, quelles que soient nos cultures nationales; il en est de même pour toutes les lois scientifiques qui constatent des phénomènes et nous permettent de les prévoir. C'est donc l'étude d'une réalité extérieure à toute culture. Mais, comme chaque culture entraîne toute une série d'expressions qu'elle a figées dans la langue au cours des siècles, il devient également évident que nous verrons cette réalité au travers d'un filtre qui s'appelle la culture. Cela influence nos hypothèses, le cheminement de nos raisonnements qui ne sont pas autre chose que des suites d'idées organisées et enchaînées logiquement. Mais, comme nous venons de le voir, chaque culture possède sa logique: n'est-ce pas Meillet qui a dit que \& chaque siècle a la grammaire de sa philosophie. Le Moyen Âge a essayé de fonder la sienne sur la logique et ... notre grammaire générale n'a été que le prolongement de la logique ${ }^{4}$. Si nous acceptons l'observation générale de Meillet, chaque culture voit sa philosophie évoluer avec le

3. Ce calque de l'anglais devrait être professions libérales.

4. A. Meillet, Introduction à l'étude comparative des langues indo-européennes, Paris, Hachette, 1921, p. 118 . 
passage du temps. Ce qui vient encore compliquer l'étude du traducteur. Mais l'évolution étant malgré tout assez lente et bien marquée par des œuvres-clés connues de tous, la recherche reste bien en-dedans des possibilités humaines.

Je me permets d'insister sur cette connaissance de la culture et je tiens à citer un exemple qui m'a frappé récemment. La traduction d'un manuel de biologie parlait évidemment de Darwin et disait que celui-ci avait effectué avec son fils, vers 1874 , des expériences sur les végétaux à l'aide de «boîtes de conserves »; or, ces dernières ne sont apparues que vers 1895 . Il y a là un anachronisme scientifique inacceptable (mais que les protestataires ont ignoré); il vient du fait que les boîtes utilisées par Darwin étaient faites de tin (fer-blanc). Le traducteur, mal informé, a traduit tin can par «boîtes de conserves». Le dictionnaire ne pouvait guère l'aider. Seule sa connaissance des réalités de la deuxième moitié du XIX siècle aurait pu l'éclairer.

Tout à l'heure, j'ai parlé du Weltanschauung ou si vous préférez, le terme, mal traduit, de «conception du monde ». Cette notion devient très importante lorsque l'on parle de l'éducation de la génération qui verra le premier homme sur la lune ou sur Mars ou Vénus, qui sait? Toute conception du monde implique une attitude préalable vis-à-vis de ce monde. Chacun se doit de poser son petit cogito, ergo sum. En pratique, chaque groupe socio-culturel le fait et cela se traduit par certaines tournures linguistiques figées que chaque génération hérite de celle qui la précède. Nous en sommes conscients mais, comme pour le problème précédent, rien n'a été systématisé. L'inventaire des syntagmes qui expriment notre engagement par rapport à l'univers qui nous entoure n'est pas encore fait; nous sommes loin encore de la systématisation qui découlera de cet inventaire. Espérons que notre attente ne sera pas trop longue. Le problème en vaut la peine. Comme preuve, je ne donnerai que ces deux phrases qui expriment la même réalité nautique: The ship runs before the wind, le bateau allait vent arrière.

Y a-t-il opposition? Le français place-t-il le locuteur au centre de l'univers? L'anglais serait-il un spectateur passif ? Voilà bien des questions dont les réponses éclaireraient notre lanterne linguistique et nous permettraient, tout en accroissant le domaine de l'art qu'est la traduction, d'en améliorer la technique.

Je m'excuse de n'avoir présenté qu'une longue série de questions auxquelles je n'ai pu répondre. Mais j'espère qu'elles entraîneront de cordiales discussions d'où jaillira la lumière. En terminant, je citerai cette phrase de $\mathrm{H}$. Palmer qui illustre si éloquemment le rapport langue-culture: «Does language come before literature and the verb to be before to be or not to be?».

Nous en sommes encore aux balbutiements de cet art qu'est la traduction, car nous avons à peine exploré la culture, nous l'avons tout au plus inventoriée, sachant très bien que cet ensemble qu'est la culture d'un peuple est le fondement à partir duquel des conséquences prévisibles pourront être envisagées et, par conséquent, des tournures linguistiques expliquées. En résumé, toute culture n'est qu'un réseau d'habitudes, et l'habitude est bien souvent la mère de la paresse et s'appelle alors routine lorsqu'elle cesse d'être le fait culturel et devient un fait technique.

Roland HaUmont 